\title{
Análisis de la generación de las irreversibilidades a partir del Coeficiente de Operación en un sistema de refrigeración por compresión mecánica de vapor con $R$ - $134 a$
}

\section{Analysis of the generation of irreversibilities from the Coefficient of Operation in a refrigeration system by mechanical vapor compression with $R-134 a$}

\author{
RANGEL-ROMERO, Carlos $\dagger^{*}$, ROJAS-GARNICA, Juan Carlos, FLORES-MARTÍNEZ, Guillermo y \\ BARCELATA-PINZÓN, Antonio
}

Universidad Tecnológica de Puebla

ID $1^{\mathrm{er}}$ Autor: Carlos, Rangel-Romero / ORC ID: 0000-0003-4879-4228, CVU CONACYT ID: 894477

ID $1^{\mathrm{er}}$ Coautor: Juan Carlos, Rojas-Garnica / ORC ID: 0000-0002-2661-587X, CVU CONACYT ID: 66417

ID $2^{\text {do }}$ Coautor: Guillermo, Flores-Martínez / ORC ID: 0000-0002-2243-2379, CVU CONACYT ID: 169853

ID $3^{\text {er }}$ Coautor: Antonio, Barcelata-Pinzón / ORC ID: 0000-0001-8287-004X, CVU CONACYT ID: 160211

\begin{abstract}
Resumen
En este trabajo se presenta el desarrollo de las contribuciones individuales, en la generación de irreversibilidades, de un sistema de refrigeración a partir del Coeficiente de Operación (COP). La generación de irreversibilidades ha sido ampliamente analizada utilizando los principios de la primera y la segunda ley de la termodinámica, dicho análisis emplea los parámetros de la entalpía, la entropía, así como la temperatura y el calor generado en cada componente. El método propuesto en este trabajo mejora la precisión de los cálculos debido al empleo del COP en el proceso matemático, el cual incluye los procesos de transferencia de calor y las caídas de presión desarrollados en el evaporador, línea de succión, compresor, línea de descarga, condensador, línea de líquido y válvula de expansión del sistema de refrigeración por compresión mecánica de vapor. Se muestran los análisis matemáticos y los resultados experimentales, con estos se concluye que el procedimiento expuesto es más cercano a las condiciones reales.
\end{abstract}

COP, Irreversibilidades, R134a

\begin{abstract}
This paper presents the development of individual contributions, in the generation of irreversibilities, of a refrigeration system based on the Coefficient of Operation (COP). The generation of irreversibilities has been widely analyzed using the principles of the first and second laws of thermodynamics. This analysis uses the parameters of enthalpy, entropy as well as the temperature and heat generated in each component. The method proposed in this work improves the accuracy of the calculations due to the use of the COP in the mathematical process, which includes the processes of heat transfer and pressure drops developed in the evaporator, suction line, compressor, discharge line, condenser, liquid line and expansion valve of the mechanical vapor compression refrigeration system. The mathematical analyzes and the experimental results are shown, with these it is concluded that the exposed procedure is closer to the real conditions than those traditional procedures found in the text books.
\end{abstract}

COP, Irreversibilities, R134a

Citación: RANGEL-ROMERO, Carlos, ROJAS-GARNICA, Juan Carlos, FLORES-MARTíNEZ, Guillermo y BARCELATA-PINZÓN, Antonio. Análisis de la generación de las irreversibilidades a partir del Coeficiente de Operación en un sistema de refrigeración por compresión mecánica de vapor con R-134a. Revista de Ingeniería Mecánica. 2019. 3-12: $10-15$

\footnotetext{
* Correspondencia al Autor (Correo electrónico: carlos.rangel@utpuebla.edu.mx)

$\dagger$ Investigador contribuyendo como primer Autor.
} 


\section{Introducción}

Actualmente los sistemas de refrigeración tienen una gran aplicación en el sector doméstico, comercial e industrial y representan una fracción importante del consumo de energía a nivel mundial por lo que es necesario hacer frente al incesante aumento de consumo de energía debido a la escasez de fuentes y recursos renovables. Un sistema de refrigeración por compresión mecánica de vapor debe estar enfocado al ahorro energético. Para tal fin debe contar con equipos eficientes, el empleo del refrigerante adecuado que contribuya a un menor calentamiento global y a la destrucción de la capa de ozono y una operación correcta de los equipos principales del sistema de refrigeración como son el evaporador, compresor, condensador y válvula de expansión.

Es por ello que se hace necesario crear una cultura de ahorro energético que permita hacer uso de los recursos existentes de la mejor forma posible. El campo de la refrigeración no es ajeno a ello. Por lo que es indispensable optimizar de la manera más eficiente posible estos sistemas.

Existen diversos parámetros que deben estar claros a la hora de realizar una evaluación a los componentes del sistema de refrigeración, por ejemplo el método más empleado para realizar una evaluación de energía al sistema de refrigeración es el cálculo de las irreversibilidades originadas en cada componente principal de los sistemas de refrigeración, y con este análisis se conoce la situación y condiciones en su real dimensión, como pueden ser, la cantidad a ahorrar y las caídas de presión en las tuberías y la transferencia de calor que existe entre el refrigerante y el medio ambiente, la fricción del refrigerante al fluir por los componentes, solo por mencionar algunas, lo cual requiere una medición o evaluación constante en los componentes para así esbozar de manera más real y exacta el consumo de energía y optimizar de esta manera los procesos termodinámicos.

En este trabajo se presenta el desarrollo de las contribuciones individuales, en la generación de irreversibilidades, de un sistema de refrigeración a partir del Coeficiente de Operación (COP).
Los factores que provocan las irreversibilidades en un sistema de refrigeración por compresión mecánica de vapor pueden ser originadas a causa de la transferencia de calor a través de una diferencia finita de temperaturas, la expansión libre, la mezcla de dos gases, entre otras más. Las causas de las irreversibilidades para nuestro estudio son la fricción, la expansión y compresión de no cuasiequilibrio y la transferencia de calor, las cuales se toman en cuenta en el desarrollo de las contribuciones individuales en la generación del COP, dentro del modelo teórico implementado a una metodología experimental la cual fue desarrollada en el Laboratorio de Ingeniería Térmica e Hidráulica Aplicada (LABINTHAP) de la SEPI-ESIME-ZACATENCO-IPN.

\section{Metodología}

En este trabajo se presenta el procedimiento que se sigue para realizar el análisis termodinámico del ciclo de refrigeración por compresión mecánica de vapor, este análisis se basa en la aplicación de la primera y segunda ley de la termodinámica para obtener las irreversibilidades generadas en el sistema de refrigeración. Una vez calculadas las irreversibilidades se hace un análisis termodinámico empleando la definición del COP y con esta aplicación se muestran las contribuciones individuales en el sistema de refrigeración y que es un parámetro importante para la evaluación de la energía. La primera ley de la termodinámica aplicada a un volumen de control describe el balance de energía mediante la ecuación:

$$
\begin{aligned}
& m_{e}\left(h+\frac{V^{2}}{2}+g z\right)-m_{S}\left(h+\frac{V^{2}}{2}+g z\right)+Q-W \\
& =\left[m_{f}\left(u+\frac{V^{2}}{2}+g z\right)-m_{\mathrm{i}}\left(u+\frac{V^{2}}{2}+g z\right)\right]
\end{aligned}
$$

Para flujo permanente y despreciando los cambios de energía cinética y potencial, el flujo de masa a la entrada y a la salida permanece constante y la variación de la energía es nula, por lo tanto, la ecuación se simplifica:

$Q-W=m\left(h_{s}-h_{e}\right)$

Esta ecuación se aplica para hacer el balance de energía del compresor, porque se le suministra potencia mecánica e intercambia calor con el exterior. 
Para el evaporador, el condensador y las diferentes tuberías a las que no se le suministra trabajo mecánico, pero intercambian calor con el exterior y por lo tanto la ecuación se escribe como:

$$
Q=m\left(h_{s}-h_{e}\right)
$$

Para la válvula de expansión a la que no se le suministra trabajo mecánico y tampoco intercambia calor con el exterior $\mathrm{Q}=0$. En función de la entropía la segunda ley de la termodinámica se expresa como:

$$
\begin{aligned}
\left(S_{f}-S_{i}\right)_{S i s t}= & \int \operatorname{rev} \frac{\delta Q}{T}+\sum(m s)_{e}- \\
& \sum(m s)_{s}+\Delta S_{i n t}
\end{aligned}
$$

Para flujo permanente, la variación de entropía en el sistema es cero, por lo tanto, la ecuación queda como:

$\Delta S_{\text {int }}=m\left(S_{s}-S_{e}\right)-\frac{Q}{T}$

El Coeficiente de Operación (COP), que se define como la carga térmica entre el flujo de energía suministrada en forma mecánica a través del compresor, el COP es un parámetro útil para evaluar el comportamiento del sistema de refrigeración, porque representa el número de unidades de refrigeración que se logra por unidad de energía suministrada, el COP se calcula mediante la siguiente ecuación:

$$
C O P=\frac{Q}{P_{\text {eléctrica }}}
$$

La potencia eléctrica consumida se calcula mediante la siguiente ecuación:

$$
P_{\text {eléctrica }}=\sqrt{3} V I \cos \varphi
$$

Combinando la ecuación (2) y (5) y empleando la definición del COP, se tiene la siguiente ecuación:

$$
\begin{aligned}
\Delta E=0= & Q_{\text {cond }}+Q_{\text {comp }}+Q_{\text {succ }}^{t u b}+Q_{\text {desc }}^{\text {tub }}- \\
& Q_{\text {evap }}-P_{\text {eléctrica }}
\end{aligned}
$$

$$
\Delta S=0=\frac{Q_{\text {cond }}+Q_{c o m p}+Q_{\text {succ }}^{t u b}+Q_{\text {des }}^{t u b}}{T_{\text {amb }}}-\frac{Q_{\text {evap }}}{T_{\text {evap }}}-
$$

$$
\begin{aligned}
\frac{1}{C O P}= & -1+\frac{T_{a m b}}{T_{\text {evap }}}+\frac{T_{a m b} \Delta S_{\text {int }}}{Q_{\text {evap }}}+ \\
& \frac{T_{a m b}\left(\Delta S_{\text {comp }}+\Delta S_{\text {Succ }}^{t u b}+\Delta S_{\text {des }}^{t u b}\right)}{Q_{\text {evap }}}
\end{aligned}
$$

Con la ecuación (10) se observa que, a partir de la definición del COP, se tienen las contribuciones individuales de las irreversibilidades y cada una de ellas representa una evaluación del comportamiento de la energía. Estas contribuciones se pueden agrupar en un COP externo que mide la contribución del refrigerante con respecto al medio ambiente.

$\frac{1}{\operatorname{COP}_{\text {ext }}}=-1+\frac{T_{\text {amb }}}{T_{\text {evap }}}$

También se puede definir un COP interno que evalúa el comportamiento entre la generación de irreversibilidad que se origina entre el condensador y el evaporador, con respecto al calor absorbido del refrigerante en el evaporador de la sustancia a enfriar, tomando en cuenta la temperatura ambiente.

$\frac{1}{\operatorname{COP}_{\text {int }}}=\frac{T_{\text {amb }} \Delta S_{\text {int }}}{Q_{\text {evap }}}$

El COP del compresor que es la suma de las irreversibilidades que se originan en el compresor, en la tubería de succión y tubería de descarga con respecto al calor absorbido del refrigerante en el evaporador del espacio a enfriar, tomando en cuenta la temperatura ambiente, se define como:

$\frac{1}{\operatorname{COP}_{\text {comp }}}=\frac{T_{a m b}\left(\Delta S_{\text {comp }}+\Delta S_{\text {succ }}^{t u b}+\Delta S_{\text {des }}^{t u b}\right)}{Q_{e v a p}}$

Este modelo teórico desarrollado es aplicado a los datos obtenidos en forma experimental. Los experimentos se realizaron para enfriar agua a un flujo en el evaporador de $1.1 \mathrm{~L} / \mathrm{s}$, utilizando como refrigerante R-134a y a una carga térmica de $4.6 \mathrm{~kW}$. Durante el desarrollo de los experimentos se tomaron lecturas de temperaturas y presiones del refrigerante en cada entrada y salida del evaporador, compresor, condensador y válvula de expansión, para el agua se verificó el flujo del agua y la temperatura del agua a la entrada y salida del evaporador, asi como la temperatura del depósito de agua, que es la sustancia a enfriar. También se midieron la temperatura ambiente y los parámetros eléctricos necesarios para calcular la potencia suministrada al compresor.

RANGEL-ROMERO, Carlos, ROJAS-GARNICA, Juan Carlos, FLORES MARTÍNEZ, Guillermo y BARCELATA-PINZÓN, Antonio. Análisis de la generación de las irreversibilidades a partir del Coeficiente de Operación en un sistema de refrigeración por compresión mecánica de vapor con R-134a. Revista de Ingeniería Mecánica. 2019 
Los ciclos reales de refrigeración por compresión mecánica de vapor difieren de los ciclos ideales, por las irreversibilidades que se generan en los diferentes equipos del sistema de refrigeración, estas irreversibilidades aunque no se pueden evitar se deben reducir a un minimo porque traen como consecuencia la necesidad del suministro de potencia adicional para lograr la refrigeración deseada ya que mientras las irreversibilidades aumenten también sucede lo mismo con la potencia suministrada al compresor. Con el cálculo de las irreversibilidades se puede apreciar que componente tiene la mayor irreversibilidad generada en el sistema de refrigeración.

\section{Análisis de Resultados}

En la figura 1 se muestra el comportamiento del Coeficiente de Operación, la suma de la distribución da como resultado el COP total, como se puede observar el que mayor contribuyó al rendimiento del ciclo de refrigeración es el COP del compresor, a pesar de que ahí se originan las mayores irreversibilidades es también ahí donde se presentó una mayor aportación para el COP total, que es un parámetro importante para el aprovechamiento de la energía. Posteriormente, sigue el COP externo que resultó de la diferencia finita de la temperatura de evaporación del refrigerante con respecto a la temperatura ambiente y el que menor aportó al valor del COP total es el COP interno. De las tres aportaciones que contribuyen al valor del COP total, en este estudio únicamente se analizaron el COP compresor y el COP interno ya que se pueden llegar a modificar o minimizar las irreversibilidades y con esto lograr un aumento en el COP total, con lo que respecta al COP externo, los valores que no se pueden controlar para mejorar su aportación al COP total son la temperatura ambiente y la temperatura de evaporación.

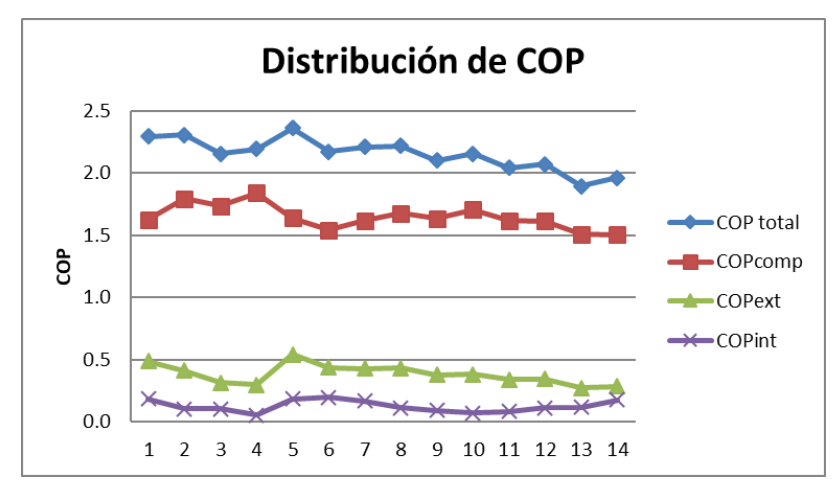

Figura 1 Distribución de aportaciones al COP total Fuente: Elaboración Propia
En la figura 2 se observa el comportamiento del COP interno con respecto a la generación de irreversibilidades, es importante resaltar que el comportamiento del COP interno fue proporcional a la generación de irreversibilidades en el condensador, este parámetro nos indicó que para aumentar el valor numérico del COP interno es necesario minimizar la irreversibilidad del componente, asimismo la irreversibilidad en el evaporador tiende a comportarse de manera uniforme.

En la figura 3 se muestra la evolución del COP compresor con respecto a la irreversibilidad total en el sistema de refrigeración y la irreversibilidad del compresor, es claro en la gráfica que el comportamiento de la irreversibilidad total presentó una tendencia igual al del compresor, con respecto al COP del compresor se observa que tiende a disminuir de valor cuando la irreversibilidad total y del compresor tienden ir en aumento.

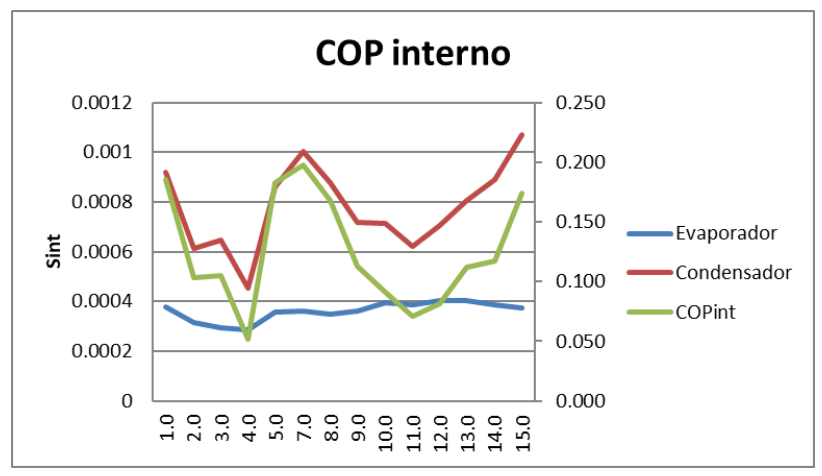

Figura 2 Comparación del COP interno con respecto a la entropía en el evaporador y condensador

Fuente: Elaboración Propia

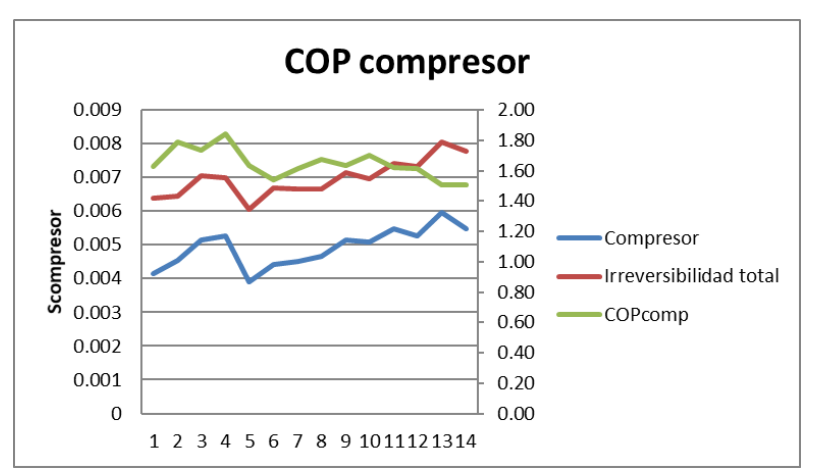

Figura 3 Comparación del COP del compresor con respecto al irreversibilidad total y la entropía del compresor

Fuente: Elaboración Propia 
En la Figura 4 se muestra como el COP interno logró disminuir de valor en cuanto la irreversibilidad total y la potencia suministrada al compresor fueron aumentando, lo que indica que el COP interno decrece lo que ocasionó que su aporte al valor del COP total disminuyó, una de las causas de este comportamiento son las caídas de presión que existen en los equipos del condensador y evaporador, asi como las tuberías que los conectan. Es importante mencionar que una caída de presión en la tuberías merma la capacidad del evaporador de absorber calor del espacio a enfriar por lo que obliga al compresor a funcionar con una menor presión de succión con el único propósito de mantener la temperatura de evaporación constante y a medida que decrece la presión de succión la potencia suministrada va en aumento y la irreversibilidad total se comporta de la misma manera ocasionando que el COP interno disminuya.

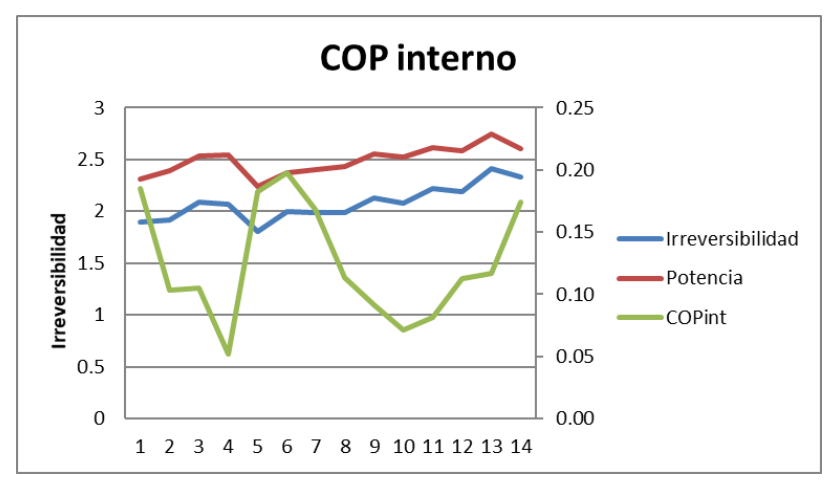

Figura 4 Comparación del COP interno con respecto al irreversibilidad total del sistema y la potencia del mismo Fuente: Elaboración Propia

En la figura 5 se muestra una relación de la irreversibilidad total con respecto a la potencia suministrada al compresor y esta relación se compara con el COP del compresor y nos muestra una tendencia que nos indica que cuando la relación de irreversibilidad total y potencia suministrada al compresor disminuyeron la COP del compresor aumentó lo que trae como consecuencia que el COP total y la eficiencia en el sistema de refrigeración presentaran el mismo comportamiento, por lo que esta tendencia nos indica que para que pueda disminuir la relación de irreversibilidad total y potencia suministrada al compresor es necesario que la transferencia de calor de la línea de succión, la línea de descarga, y en el compresor, asi como también las caídas de presión tienen que disminuir para que esta relación disminuya y el COP de compresor aumente y de esta forma mejore el proceso del sistema de refrigeración.
Es importante mencionar que la caída de presión debida a la fricción en la línea de succión no debe provocar un cambio excesivo en la temperatura de saturación, ya que origina la degradación de la energía aumente y esto ocasiona una disminución del COP del compresor.

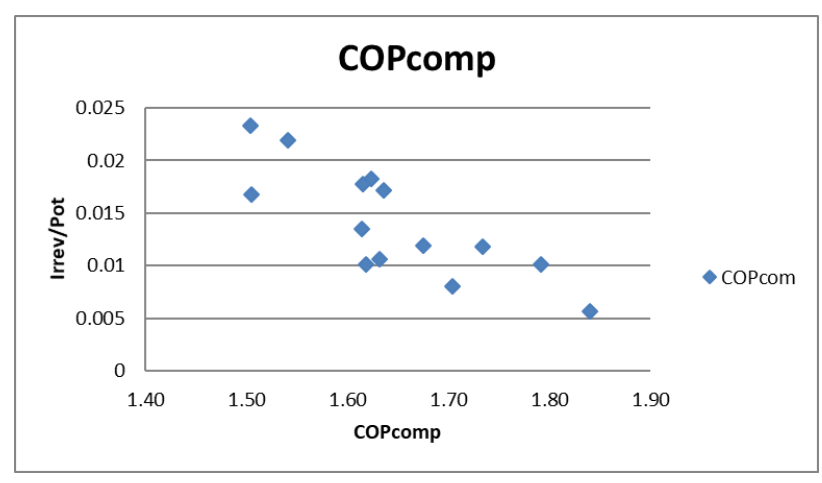

Figura 5 Comportamiento del COP del compresor con respecto al cociente de la irreversibilidad entre la potencia del sistema

Fuente: Elaboración Propia

En la figura 6 se observa como el COP interno tendió a aumentar cuando la relación de la irreversibilidad total y la potencia suministrada al compresor aumentaron, lo que trae como consecuencia que para mejorar el parámetro del COP interno es necesario que la producción de irreversibilidad aumente, lo que afecta al COP del compresor.

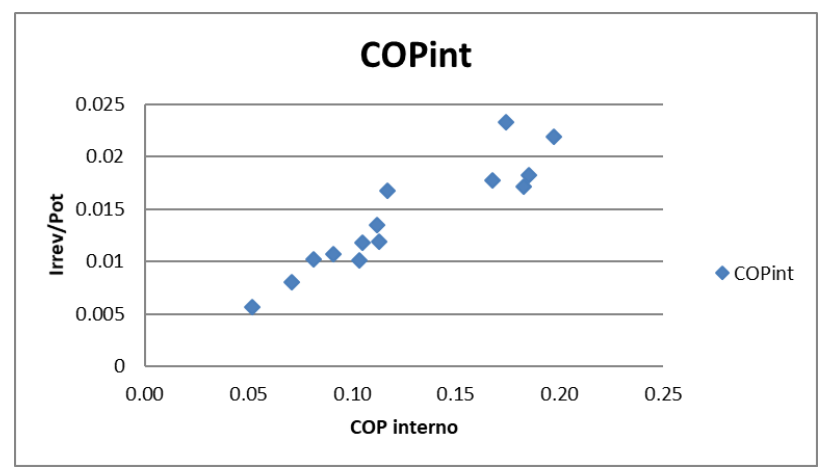

Figura 6 Comportamiento del COP interno con respecto al cociente de la irreversibilidad entre la potencia del sistema

Fuente: Elaboración Propia

\section{Agradecimientos}

Se agradece las facilidades prestadas para la elaboración del presente trabajo a la Universidad Tecnológica de Puebla y al Instituto Politécnico Nacional. 


\section{Conclusiones}

En este trabajo se muestra que el estudio de la conversión y el uso eficiente de la energía para emplear los sistemas de refrigeración por compresión mecánica de vapor es importante. El cálculo de las irreversibilidades a partir del Coeficiente de Operación tiene como propósito representar de una manera más clara cómo también las irreversibilidades aportan al uso eficiente de la energía, mediante una distribución de las irreversibilidades a través del COP total. Con este modelo desarrollado se muestra que para obtener un mejor COP total es necesario aumentar el valor del COP del compresor, lo cual se logra minimizando la entropía generada en el ciclo en función de la relación de temperaturas del fluido de trabajo con respecto a la temperatura del medio ambiente. Con esta aportación se considera que se puede mejorar el rendimiento de un sistema de refrigeración.

\section{Trabajo Futuro}

Se propone realizar un estudio para encontrar un punto de operación óptimo mediante la distribución de las irreversibilidades empleando el COP del compresor, el COP interno y el COP externo a una misma carga térmica.

\section{Referencias}

Beenon W. L., Pham H. M. (2003). Vaporinjected scroll compressor. ASHRAE Journal, 45(4): Pp. 22-27.

Cho H, Chung J. T., Kim Y. (2003). Influence of liquid refrigerant injection on the performance of an inverter-driven scroll compressor. International Journal of Refrigeration, 26: Pp. 87-94.

Di Nicola G., Brandoni C., Di Nicola C., Giuliani G. (2012). Triple point measurements for alternative refrigerants. J. Therm. Anal. Calorim.,108: Pp. 627-631.

Ding Y J, Chai Q H, Ma G Y, et al. (2004). Experimental study of an improved air source heat pump. Energy Conversion and Management, 45: Pp. 2393-2403.
Dutta A., Yanagisawa T., Fukuta M. (2001). An investigation of the performance of a scroll compressor under liquid refrigerant injection. International Journal of Refrigeration, 24: Pp. 577-587.

Fukuda S., Kondou C., Takata N., Koyama S. (2013). Low GWP refrigerants R1234ze(E) and $\mathrm{R} 1234 z e(Z)$ for high temperature heat pumps. Int. J. Refrig., 40: Pp. 161-173.

Kondo S., Takizawa K., TokuhashK. I. (2012). Effects of temperature and humidity on the flammability limits of several $2 \mathrm{~L}$ refrigerants. J. Fluor. Chem., 144: Pp. 130-136.

Navarro E., Redon A., Gonzalvez-Macia J., Martinez-Galvan I. O., Corberan J. M. (2013). Characterization of a vapor injection scroll compressor as a function of low, intermediate and high pressures and temperature conditions. International Journal of Refrigeration, 36: Pp. 1821-1829.

Qiu G., Meng X., Wu J. (2013). Density measurements for 2,3,3,3-tetrafluoroprop-1-ene (R1234yf) and trans- 1,3,3,3-tetrafluoropropene (R1234ze(E)). J. Chem. Thermodyn., 60: Pp. 150-158.

X Meng., Qiu G., Wu J., Abdulagato I. M. (2013). Viscosity measurements for 2,3,3,3tetrafluoroprop-1-ene (R1234yf) and trans1,3,3,3-tetrafluoropropene (R1234ze(E)). J. Chem. Thermodyn., 63: Pp. 24-30.

Xu S. X., Ma G. Y. (2015). Working performance of R-32 two-stage compression system in domestic air-conditioner. Energy and Buildings, 93: Pp. 324-331.

Yang M., Wang B. L., Li X. T., Shi W. X., Zhang L. (2015). Evaluation of two-phase suction, liquid injection and two-phase injection for decreasing the discharge temperature of the R32 scroll compressor. International Journal of Refrigeration, 59: Pp. 269-280. 